Review article

\title{
Thyroid autoimmunity in bipolar disorder: A systematic review
}

\author{
Margherita Barbuti ${ }^{\mathrm{a}, \mathrm{b}}$, André F. Carvalho ${ }^{\mathrm{c}}$, Cristiano A. Köhler ${ }^{\mathrm{c}}$, Andrea Murru ${ }^{\mathrm{a}}$, \\ Norma Verdolini $^{\mathrm{a}, \mathrm{d}}$, Giovanni Guiso ${ }^{\mathrm{a}}$, Ludovic Samalin ${ }^{\mathrm{e}}$, Michael Maes ${ }^{\mathrm{f}, \mathrm{g}, \mathrm{h}, \mathrm{i}, \mathrm{j}}$, Brendon Stubbs ${ }^{\mathrm{k}, 1}$, \\ Giulio Perugi $^{\mathrm{b}}$, Eduard Vieta ${ }^{\mathrm{a}, *}$, Isabella Pacchiarotti ${ }^{\mathrm{a}}$ \\ a Bipolar Disorders Unit, Clinical Institute of Neurosciences, Hospital Clinic, IDIBAPS, University of Barcelona, CIBERSAM, Barcelona, Catalonia, Spain \\ b University of Pisa, Pisa, Italy \\ c Translational Psychiatry Research Group and Department of Clinical Medicine, Faculty of Medicine, Federal University of Ceara, Fortaleza, CE, Brazil \\ d Division of Psychiatry, Clinical Psychology and Rehabilitation, Department of Medicine, Santa Maria della Misericordia Hospital, University of Perugia, Perugia, Italy \\ e CHU Clermont-Ferrand, Department of Psychiatry, EA 7280, University of Auvergne, Clermont-Ferrand, France \\ ${ }^{\mathrm{f}}$ Department of Psychiatry, Faculty of Medicine, Chulalongkorn University, Bangkok, Thailand \\ g IMPACT Strategic Research Center, School of Medicine, Barwon Health, Deakin University, Geelong, Australia \\ ${ }^{\mathrm{h}}$ Health Sciences Postgraduate Program, Health Sciences Center, State University of Londrina, Londrina, Brazil \\ ${ }^{\mathrm{i}}$ Revitalis, Waalre, The Netherlands \\ j Department of Psychiatry, Medical University of Plovdiv, Plovdiv, Bulgaria \\ k Physiotherapy Department, South London and Maudsley NHS Foundation Trust, Denmark Hill, London SE5 8AZ, United Kingdom \\ ${ }^{1}$ Health Service and Population Research Department, Institute of Psychiatry, Psychology and Neuroscience, King's College London, De Crespigny Park, Box SE5 8AF, \\ London, United Kingdom
}

\section{A R T I C L E I N F O}

\section{Keywords:}

Bipolar disorder

Thyroid autoimmunity

Lithium treatment

Thyroid autoantibodies

\begin{abstract}
A B S T R A C T
Background: Accumulating evidence points to the pathophysiological relevance between immune dysfunction and mood disorders. High rates of thyroid dysfunction have been found in patients with bipolar disorder (BD), compared to the general population. A systematic review of the relationship between BD and thyroid autoimmunity was performed.

Methods: Pubmed, EMBASE and PsycINFO databases were searched up till January 28th, 2017. This review has been conducted according to the PRISMA statements. Observational studies clearly reporting data among BD patients and the frequency of autoimmune thyroid pathologies were included.

Results: 11 original studies met inclusion criteria out of 340 titles first returned from the global search. There is evidence of increased prevalence of circulating thyroid autoantibodies in depressed and mixed BD patients, while there is no evidence showing a positive relationship between BD and specific autoimmune thyroid diseases. There is a controversy about the influence of lithium exposure on circulating thyroid autoantibodies, even if most of studies seem not to support this association. A study conducted on bipolar twins suggests that autoimmune thyroiditis is related to the genetic vulnerability to develop BD rather than to the disease process itself. Females are more likely to develop thyroid autoimmunity.

Limitations: The samples, study design and outcomes were heterogeneous.

Conclusion: Thyroid autoimmunity has been suggested to be an independent risk factor for bipolar disorder with no clear association with lithium exposure and it might serve as an endophenotype for BD.
\end{abstract}

\section{Introduction}

Recent emergent evidence points to the importance of the etiopathogenetic relationship between activation of immune-inflammatory pathways and psychiatric syndromes, including bipolar disorder (BD) (Berk et al., 2011; Grande et al., 2016; Hamdani et al., 2013; Leon-Caballero et al., 2015; Maes et al., 1995; Rege and Hodgkinson, 2013; Siwek et al., 2016). Available data support a relationship between autoimmunity and
BD. For example, BD patients have significantly more and higher autoantibodies (autoAbs) compared with healthy controls and other psychiatric patients (Sidhom et al., 2012). A large cohort study reported that nonspecific autoimmune processes could precede the onset of $\mathrm{BD}$ (Eaton et al., 2010). Furthermore, it is quite known that certain autoimmune diseases, such as the neuropsychiatric systemic lupus erythematosus, Hashimoto's thyroiditis and multiple sclerosis are often associated with symptoms of mania and depression (Carta et al., 2014; Rege and Hodgkinson, 2013).

\footnotetext{
* Correspondence to: Bipolar Disorders Unit, Hospital Clínic, IDIBAPS, CIBERSAM, C/ Villaroel 170, 08036 Barcelona, ES, Spain.

E-mail address: EVIETA@clinic.cat (E. Vieta).
} 
The association between thyroid alterations and mood disorders has been known for some time (Whybrow et al., 1969). It has been suggested an implication of hypothalamic-pituitary-thyroid (HPT) axis on neuropsychological deficits of BD (Bonnin et al., 2010). High rates of thyroid dysfunction have been found in patients with $\mathrm{BD}$, compared to the general population (Goodwin and Jamison, 2007). Many studies show a possible relationship between circulating thyroid autoAbs, including anti-thyroperoxidase (TPO-Abs), and affective disorders even in the absence of thyroid hormone abnormalities (Leyhe and Mussig, 2014). A higher prevalence of thyroid autoAbs was found among patients with BD, even in the absence of thyroiditis (Kupka and Regeer, 2007). Thus, thyroid alterations may play a role in the pathophysiology of $\mathrm{BD}$, although their exact role, if any, has not been fully elucidated. Pharmacological treatment, especially lithium, which is considered the mainstay of BD treatment, might be also implicated as a predisposing factor for the development of autoimmune thyroiditis (AIT) (Chakrabarti, 2011). Furthermore, it is widely recognized that two important factors, age and sex, may influence the incidence of thyroid autoimmunity: middle-aged women are known to show the highest prevalence rates (Bocchetta and Loviselli, 2006).

In autoimmune thyroid disease, increased levels of autoAbs against thyroperoxidase (TPO-Abs), previously named anti-microsomal (MAbs), against thyroglobulin (TG-Abs) and against thyroid-stimulatinghormone-receptors (TRAbs) are commonly found (Nielsen et al., 2004). The prevalence of TPO-Abs seems to be higher in autoimmune hypothyroidism and Grave's disease (GD). The positivity for TG-Abs has been found in less than $60 \%$ of patients with lymphocytic thyroiditis and $30 \%$ of GD (Silva et al., 2003). TRAbs were found to be increased in GD and atrophic thyroiditis (Boelaert and Franklyn, 2005).

The major aims of the present systematic review are to better elucidate the relationship between BD and thyroid autoimmunity and the possible evidence of a primary autoimmune thyroid response in $\mathrm{BD}$ patients. Also, we aimed at reviewing the role of lithium treatment and sex in the prevalence of thyroid autoimmunity among BD patients. Finally we will discuss the potential research implications of our findings for future studies.

\section{Method}

This review has been conducted according to the Preferred Reporting Items for Systematic Reviews and Meta-Analyses (PRISMA) statements (Liberati et al., 2009) (see Fig. 1).

\subsection{Search strategy and selection of studies}

Studies were identified through three methods. First, a comprehensive computerized literature search in three bibliographical databases - MEDLINE, EMBASE and PsycInfo - from inception to January 28th, 2017 was performed. Search strings are provided in the Supplementary material S1 that accompanies the online edition of this article. Second, this search strategy was augmented through tracking citations of included articles in Google Scholar. Finally, we conducted a manual reference check of accepted papers to supplement the above electronic searches (see references of studies excluded after full-text review in the Supplementary material S2). No language restrictions were applied. Two investigators screened title/abstracts for potential eligibility. Disagreements were resolved through consensus. References selected for full-text review were evaluated by two independent raters. Disagreements were resolved by discussion. When there were overlapping samples in different studies, we included the one with the largest dataset. We included observational studies (cross-sectional, case-control and cohort studies) in which data on patients meeting either DSM or ICD criteria for BD and frequency or incidence of autoimmune thyroid pathologies (or frequency of positive thyroid autoAbs or as a continuous measure) were clearly reported. We also included studies investigating the prevalence of autoimmune thyroid pathology

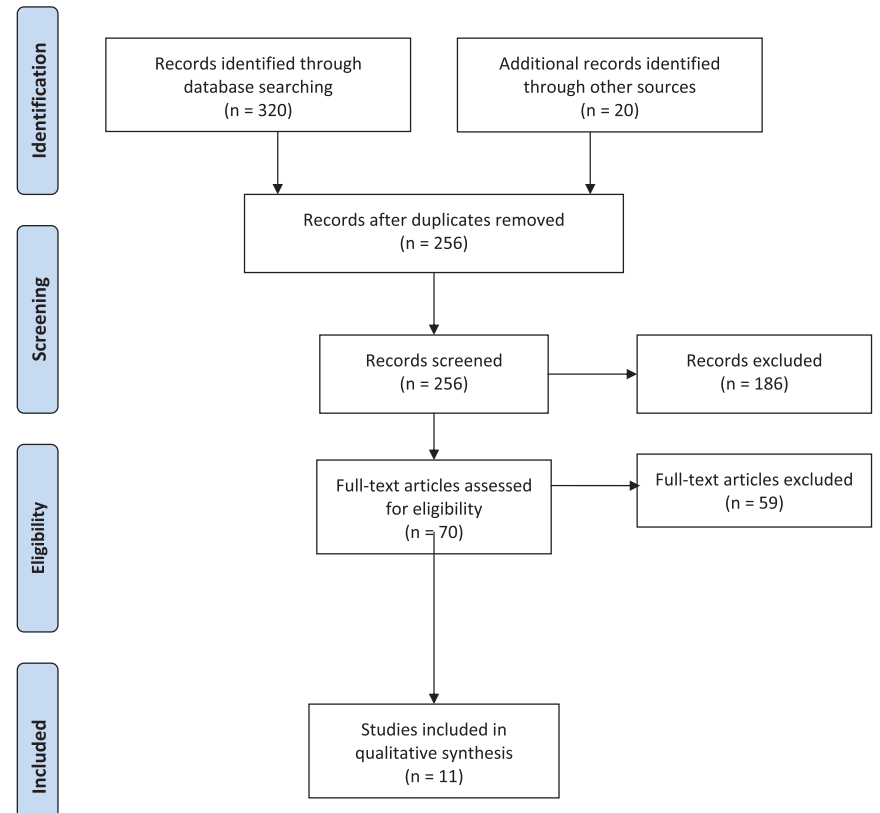

Fig. 1. PRISMA flow diagram of the selected and included studies.

or thyroid autoAbs (positivity or continuous measure if compared to controls) in unaffected first-degree relatives or unaffected twins of individuals with BD. Studies were excluded if they: involved less than ten bipolar patients; reported other psychiatric disorders, and did not provide data for BD separately; reported thyroid function assays without autoAbs; reported thyroid pathology but did not specify if these conditions were autoimmune.

\subsection{Data extraction and quality assessment}

Using a structured spread sheet, data on the following characteristics were extracted: author, publication year, study design, sample size, autoimmune thyroid pathology and anti-thyroid antibodies. We appraised the quality of included studies by using the NewcastleOttawa Quality Assessment Scale (NOS) (Wells et al., 2014) in which a study is judged on three broad perspectives: the selection of the study groups; the comparability of the groups; the ascertainment of either the exposure or outcome of interest. Two authors extracted data and assessed each included study according to the NOS criteria (See Supplementary material, Table 1). Disagreements were resolved through consensus.

\subsection{Synthesis of results}

Due to the anticipate heterogeneity in terms of study design, participants and outcomes, we conducted a narrative synthesis of the available studies.

\section{Results}

\subsection{Systematic search results}

The global search first returned 340 titles, and 256 were available after the removal of duplicates. We selected 70 studies to assess the fulltext. Subsequently, we excluded 59 of the retrieved articles (reasons for exclusion are provided in Supplementary material, Table 2). A total of 11 original studies met our inclusion criteria (Barbero et al., 2014; Bartalena et al., 1990; Cobo et al., 2015; Eaton et al., 2010; Haggerty et al., 1990, 1997; Hillegers et al., 2007; Hornig et al., 1999; Kraszewska et al., 2015; Kupka et al., 2002; Vonk et al., 2007). 


\subsection{Content results}

\subsubsection{Prevalence of autoimmune thyroiditis in $B D$ patients}

The only two studies specifically assessing the relationship between $\mathrm{BD}$ and a diagnosis of AIT (defined as altered thyroid autoAbs and altered TSH) found no statistically significant differences in prevalence of this disease among the three considered groups (BD I patients, their first-degree relatives and control group) (Cobo et al., 2015) and among BD, schizophrenic patients and controls (Eaton et al., 2010).

\subsubsection{Prevalence of circulating thyroid autoAbs in $B D$ patients}

Several studies assessed the prevalence of circulating thyroid autoAbs in psychiatric populations, especially among patients with mood disorders. This prevalence varies largely depending on the sensitivity and specificity of the assessment methodology used. Moreover, some studies have assessed antibodies concentrations as a continuous variable rather than the positive/negative dichotomy (See Table 1).

Haggerty et al. (1990) investigated the frequency of M-Abs and TGAbs in 173 consecutively admitted psychiatric inpatients, showing that the overall frequency of positive thyroid autoAbs titers in patients with affective disorder (9\%) did not differ from that in patients with nonaffective disorders (10\%). However, among BD patients, those mixed or depressed had higher rates of positive thyroid autoAbs titers than other psychiatric patients (See Table 2). In a similar study (Haggerty et al., 1997), the same authors confirmed a trend toward an increased prevalence of thyroid autoAbs in BD patients, but only mixed $(19 \%)$ or depressed $(16 \%)$ compared to other psychiatric patients and to control group. Kupka et al. (2002) assessed the prevalence of circulating TPOAbs in 226 outpatients with BD, 252 control subjects, and 3190 inpatients with any psychiatric diagnosis, showing that TPO-Abs were significantly more prevalent (28\%) in BD than in the other two groups (3-18\%). Conversely, Bartalena et al. (1990) studied the thyroid autoimmunity in a small population of $22 \mathrm{BD}$ women (11 with rapid cycling and 11 without rapid cycling), in comparison to a control group of 11 euthyroid women with no present or past history of psychiatric diseases and matched for age, showing similar rates of TPO-Abs and/or TG-Abs positivity in both groups (9.1\%). Hornig et al. (1999) assessed 79 BD-I patients, 24 BD-II patients and 46 unipolar depressed patients along with 22 healthy controls for the presence of thyroid autoAbs (MAbs and TG-Abs), using antibodies concentrations as a continuous variable (log-transformed titers). Contrary to prior reports, although the authors found that the effect of depressive affective state on M-Abs, but not on TG-Abs, approached statistical significance $(\mathrm{p}=0.061$ ), they did not find a significant association between any of the autoAbs and affective subtype, mood state, or psychotropic medication. In the study of Kraszewska et al. (2015), abnormally high levels of TPO-Abs and TGAbs were found in a significant proportion of BD patients (respectively $45 \%$ and $65 \%$ ). No associations were found between the duration of lithium treatment and thyroid dysfunction. Noteworthy, in this crosssectional study there was no control group. Barbero et al. (2014) conducted a cohort study to investigate whether thyroid autoimmune status was associated with a greater risk of readmission at one year of follow-up in a sample of $77 \mathrm{BD}$ patients and found that TG-Abs positivity was associated with a lower risk of rehospitalization. In this sample of BD patients, TPO-Abs were present in 39\% and TG-Abs in about $36 \%$. In the above mentioned study of Cobo et al. (2015), authors found no differences in terms of positivization of thyroid autoAbs between $\mathrm{BD}$ patients, their first-degree relatives and healthy controls.

\subsubsection{Prevalence of circulating thyroid autoAbs in $B D$ relatives and twin studies}

Hillegers et al. (2007) investigated the prevalence of autoimmune thyroiditis (TPO-Abs $>25 \mathrm{U} / \mathrm{mL}$ ) in 126 children of BD patients and 129 high school and young adult control subjects, showing a higher prevalence of TPO-Abs in bipolar offspring (8.7\%) compared to healthy controls (3.1\%), with higher statistical significance when considering 


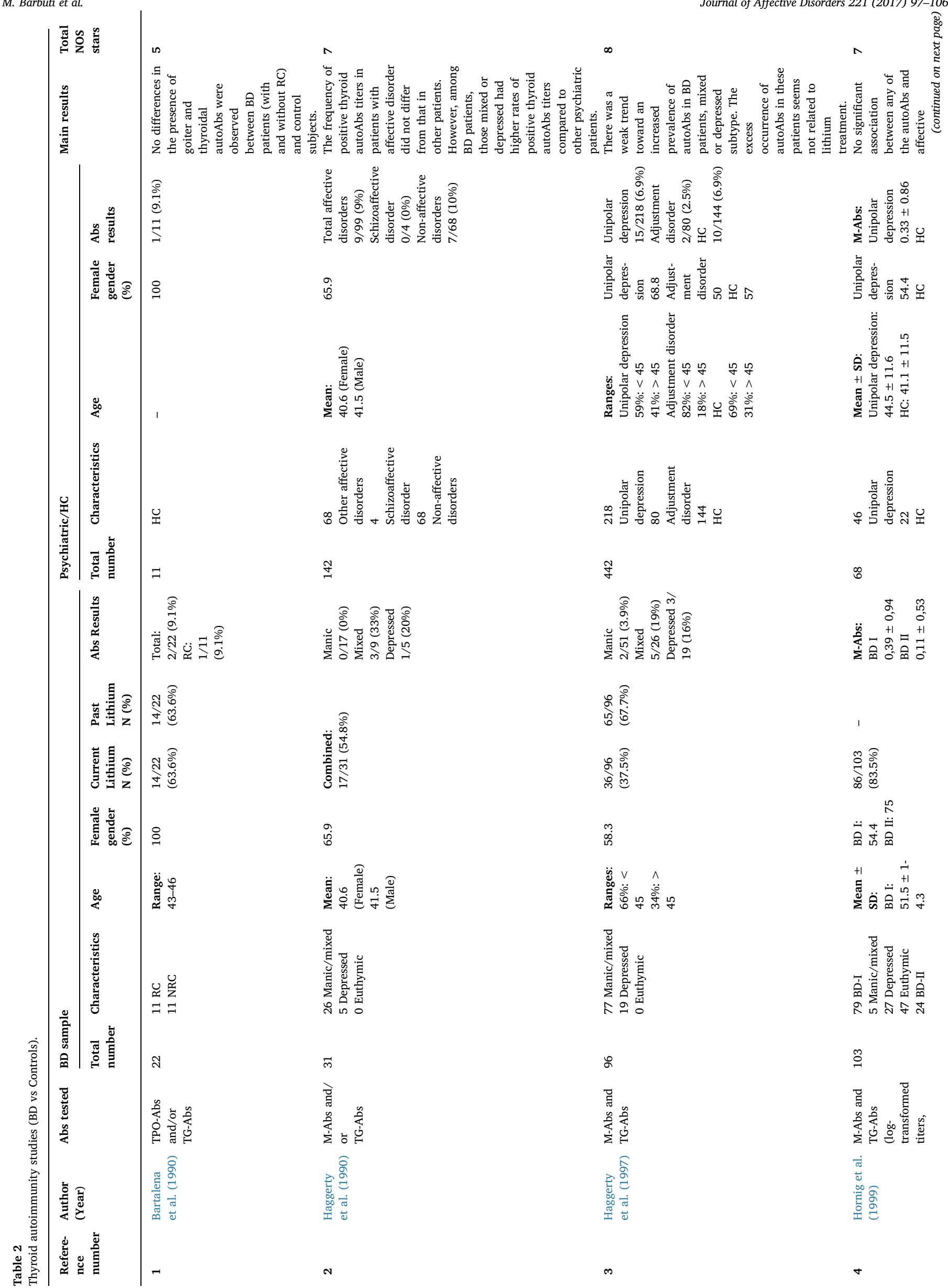




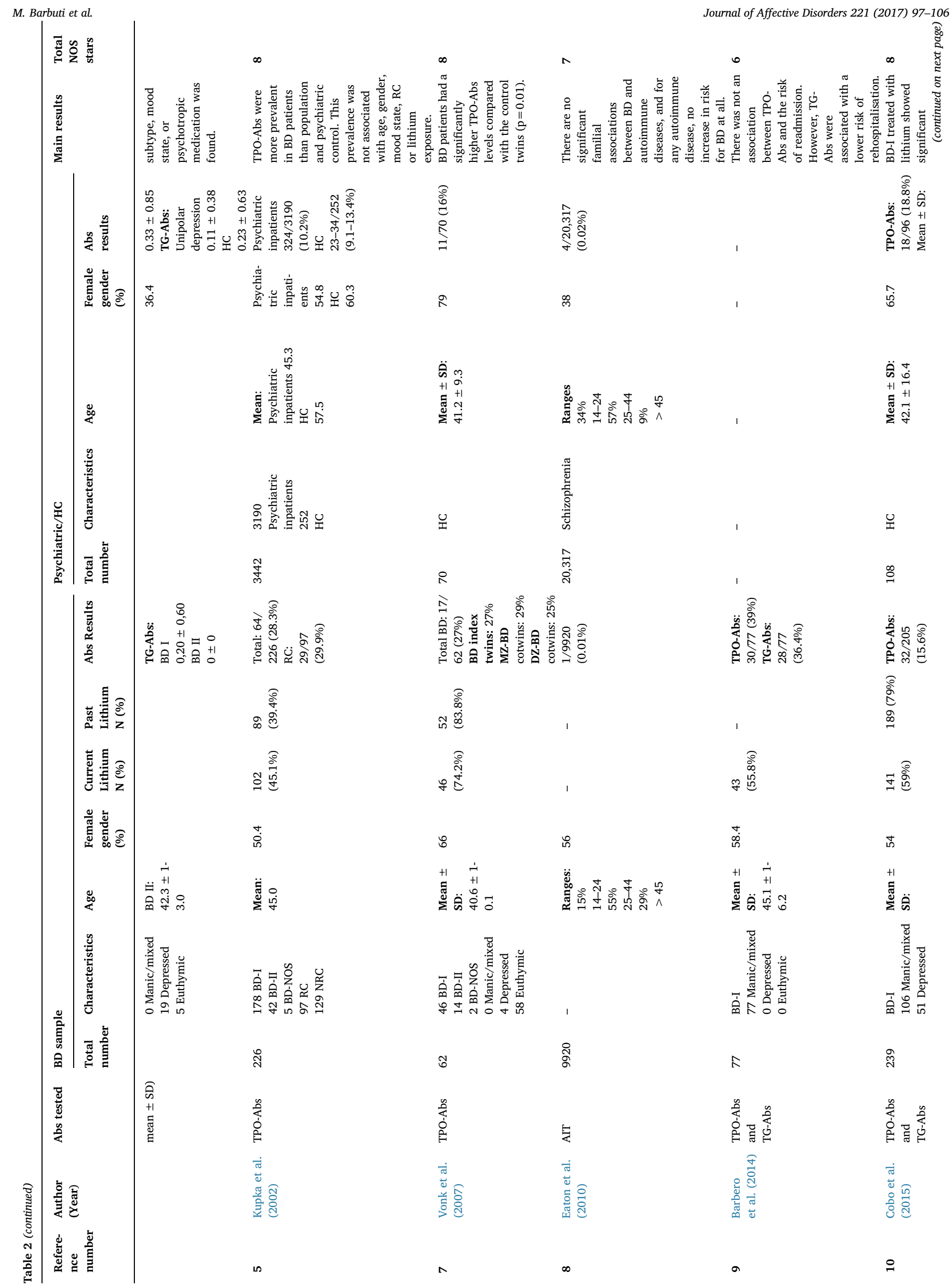




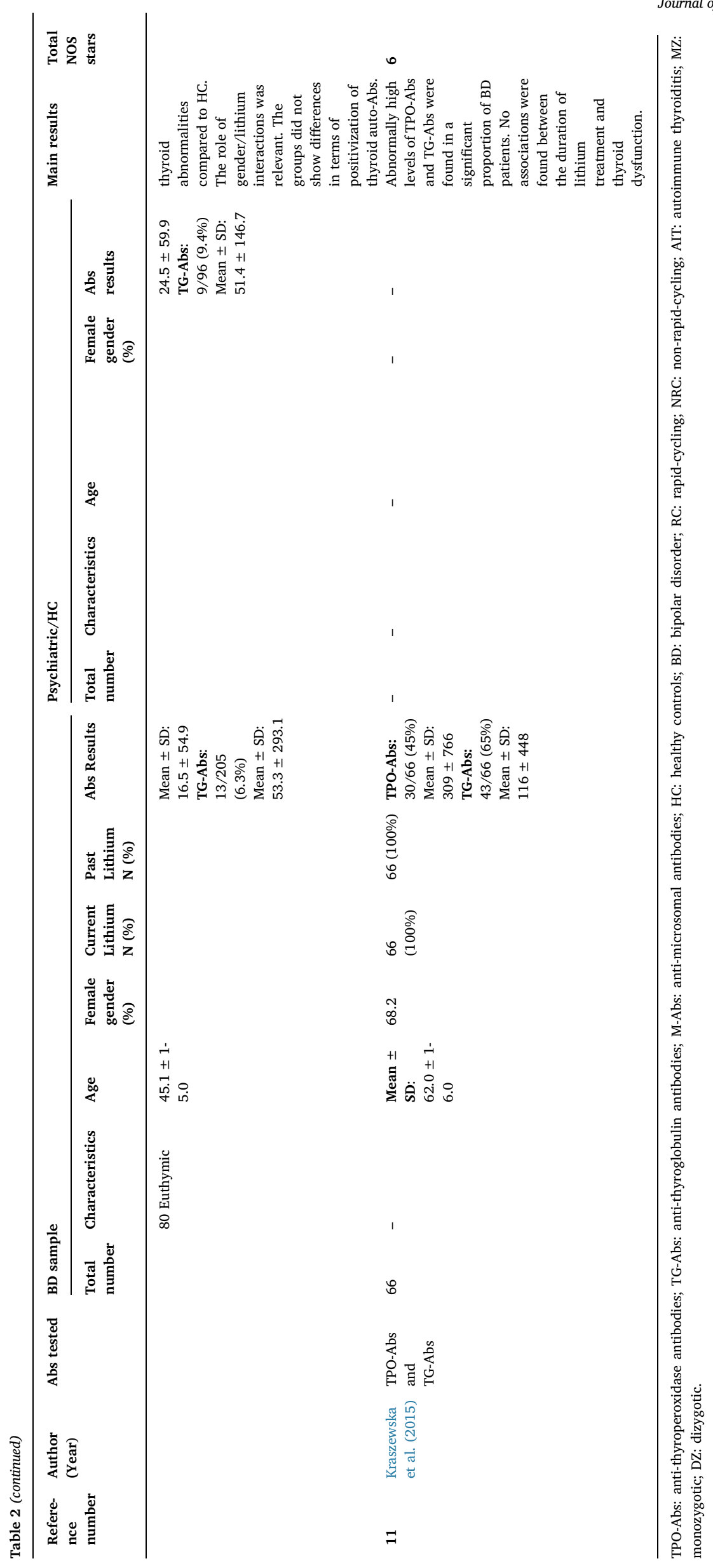




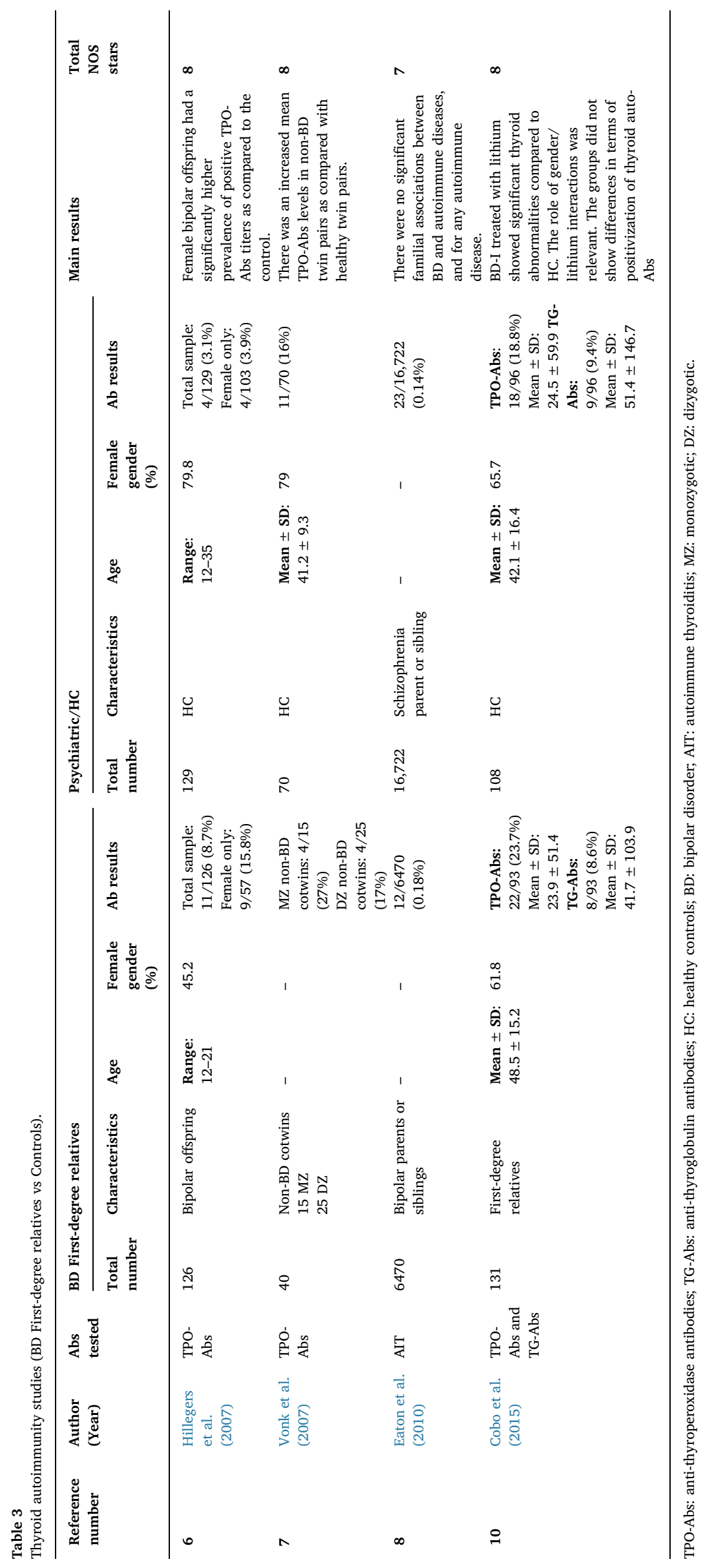


only female subjects. Therefore, children of parents with bipolar disorder were found to be more vulnerable to develop thyroid autoimmunity, independently of their vulnerability to develop psychiatric disorders (see Table 3).

Vonk et al. (2007) compared the prevalence of TPO-Abs among 22 monozygotic and 29 dizygotic twins with $\mathrm{BD}$ and 35 healthy matched control twins. This study confirmed significantly higher TPO-Abs in BD patients vs control twins ( $\mathrm{p}=0.01$ ), with TPO-Abs levels in the (discordant) non-BD cotwins between those of the $\mathrm{BD}$ patients and the control twins. The authors concluded that AIT is related not only to BD itself but also to the genetic vulnerability to develop the disorder, thus thyroid autoimmunity might represent an endophenotype for BD.

In the study of Cobo et al. (2015) BD I patients showed significant higher rates of thyroid dysfunction (i.e. subclinical hypothyroidism) compared to the other groups but no significant differences were found in terms of positivization of thyroid autoAbs. These results do not support the endophenotype theory and emphasize the positive relationship between thyroid abnormalities and sex/lithium exposure, rather than a common genetic background.

\subsubsection{Thyroid autoimmunity and lithium treatment}

Thyroid dysfunction, most commonly goiter and/or hypothyroidism, is one of the best known side effects of long-term lithium treatment (Bocchetta and Loviselli, 2006).

Kupka et al. (2002) found no significant differences between BD patients who were currently, formerly or never exposed to lithium treatment. Interestingly, the 35 (34.3\%) BD patients who had never received lithium had the highest rate of TPO-Abs. Thus, the authors concluded that the significant higher prevalence of circulating TPO-Abs in BD patients compared to healthy controls and psychiatric controls was not associated with prior lithium exposure (See Table 2).

Similar results were found in the study of Kraszewska et al. (2015), suggesting that lithium exposure could not explain the increased occurrence of thyroid autoAbs in BD patients. However, the authors did not investigate baseline levels of autoAbs (treatment naïve BD patients), while the absence of a control group is a limitation of the study. Similarly, Haggerty et al., $(1990,1997)$ found that the higher frequency of M-Abs and TG-Abs in patients with either mixed or depressed BD compared with manic BD patients was not related to lithium treatment. Bartalena et al. (1990) found that, even if lithium administration was associated to a relatively high prevalence of subclinical hypothyroidism in $\mathrm{BD}$ patients, no differences emerged in the prevalence of lithiuminduced hypothyroidism in BD patients with and without rapid cycling course. Similarly, Hornig et al. (1999) failed to demonstrate any effect of lithium treatment on thyroid autoAbs. Conversely, Cobo et al. (2015) found a positive association between lithium exposure and AIT in BD. In this study, BD-I women treated with lithium showed significant differences in thyroid function abnormalities in comparison to bipolar women who were not treated with lithium. Barbero et al. (2014) found an association between the presence of TG-Abs (but not TPO-Abs) and a lower risk of rehospitalisation at one year of follow-up and this association remained significant even after controlling for covariates such as sex, age, severity of index episode, thyroid function, duration of admission, lithium treatment, substance use and treatment adherence.

\subsubsection{The role of sex in thyroid autoimmunity of $B D$}

In the studies of Haggerty et al. $(1990,1997)$ the difference in antibodies frequency between mixed or depressed BD patients and the rest of the sample was not influenced by age and sex. Similarly, Hornig et al. (1999) found that age, sex, diagnosis and medication status could not explain the variance in thyroid autoAbs. Kupka et al. (2002) also showed that the presence of TPO-Abs in BD patients was not associated with age, sex, mood state, rapid-cycling course or lithium exposure. Kraszewska et al. (2015) found no differences between male and female lithium-treated patients in TPO-Abs and TRAbs. Female subjects had higher TG-Abs prevalence than male patients, but this difference was not statistically significant. Concentrations of TG-Abs significantly correlated with the duration of illness but there was no significant correlation with the age of the patients. Conversely, Hillegers et al. (2007) showed that female bipolar offspring had a significantly higher prevalence of positive TPO-Ab titers (15.8\%) as compared to the female controls (3.9\%) ( $\mathrm{p}=0.008)$. Vonk et al. (2007) showed a clear sex effect on the higher prevalence of AIT found in female bipolar patients compared to female control subjects ( $37 \%$ vs. $18 \%$; $p=0.02$ ) but not in male bipolar patients versus male control subjects ( $10 \%$ vs. $6 \%)$. Cobo et al. (2015) found that levels of TG-Abs and TPO-Abs were not affected by age, while the presence of thyroid autoAbs was influenced by sex, with a higher prevalence in women.

\subsubsection{Thyroid autoimmunity and rapid-cycling $B D$}

It has been suggested that $\mathrm{BD}$ with rapid-cycling course is associated with thyroid dysfunction, especially with either overtly or subclinical hypothyroidism (Bauer and Whybrow, 1986; Cho et al., 1979; Wehr and Goodwin, 1979).

However, in the study of Bartalena et al. (1990), authors did not find any differences in the prevalence of subclinical hypothyroidism, goiter and thyroid autoAbs among rapid and non-rapid cycling BD subjects. Similarly, Kupka et al. (2002) confirmed this results, showing no significant differences between rapid and non-rapid cycling BD patients.

\section{Discussion}

The first major result of our review is that there is no clear association between autoimmune thyroid dysfunction with BD. In fact, we detected a wide heterogeneity in the study samples regarding age, male-female ratio, medication status and selection of BD patients and control groups (See Table 2). We also found a large variability in the assessment of antibodies, in the units of measurement and in the threshold levels in defining positivity (see Table 1). Moreover, only few studies assessed the levels of thyroid autoAbs as a continuous measure (Cobo et al., 2015; Hornig et al., 1999; Kraszewska et al., 2015), while most studies investigated positivization as a dichotomous variable (Barbero et al., 2014; Bartalena et al., 1990; Haggerty et al., 1990, 1997; Kupka et al., 2002; Vonk et al., 2007). Regarding the diagnosis of $\mathrm{BD}$, this review included only studies in which the diagnosis was made according to DSM or ICD criteria even if a structured interview was conducted only in few studies (Cobo et al., 2015; Hillegers et al., 2007; Hornig et al., 1999; Kraszewska et al., 2015; Kupka et al., 2002; Vonk et al., 2007).

To date, there is no evidence of a positive relationship between $\mathrm{BD}$ and autoimmune thyroid diseases (Bocchetta et al., 2016). Eaton et al. (2010) found no significant familial association between BD and autoimmune diseases and in particular AIT was found in a very low percentage of BD patients $(0.01 \%)$. Furthermore, the criteria for autoimmune thyroid pathology vary widely among the studies. Despite using the term autoimmune thyroiditis, most of the studies focused on the mere presence of circulating autoAbs. The most accurate case definition for AIT seems to be the one from Cobo et al. (2015) who considered not only the positivization of thyroid autoAbs but also the altered TSH levels.

Nevertheless, there is a clear evidence of increased prevalence of circulating thyroid autoAbs in BD. Several authors suggested a potential association between bipolar spectrum disorders and circulating thyroid autoAbs reporting increased prevalence of M-Abs (TPO-Abs) and/or TG-Abs in specific subgroups of BD patients compared with healthy individuals (Haggerty et al., 1990, 1997; Hornig et al., 1999; Kupka et al., 2002; Vonk et al., 2007).

Although a study conducted in the offspring of bipolar subjects suggested that the vulnerability to present increased thyroid autoAbs was independent from the vulnerability to develop psychiatric disorders (Hillegers et al., 2007), authors concluded that the different penetrance 
of BD and AIT in the offspring was likely due to the co-effect of other inheritable influences and to different environmental factors. Recent findings showing the high rate of concordance in presenting AIT in monozygotic and dyzygotic bipolar twins (Vonk et al., 2007), could suggest an autoimmune thyroid process as a possible endophenotype for BD.

The relationship between phase and course of $\mathrm{BD}$ and autoimmune thyroid dysfunction is also under investigation. There is some evidence pointing to a higher prevalence of thyroid autoAbs during depressed and mixed states of BD patients (Haggerty et al., 1990, 1997), although not all authors could establish such an association (Hornig et al., 1999; Kupka et al., 2002).

The association between BD with rapid cycling course and thyroid dysfunction, especially hypothyroidism, has been supported by several authors (Azorin et al., 2008; Cowdry et al., 1983; Oomen et al., 1996). Regarding circulating thyroid autoAbs, one study - not included in our review

because the relationship between $\mathrm{BD}$ and thyroid autoimmunity was not the primary objective - on a large bipolar sample, found an association between increased circulating TPO-Abs and rapid cycling BD (Oomen et al., 1996), but this positive relationship has not been confirmed in other studies (Bartalena et al., 1990; Kupka et al., 2002).

Lithium exposure is another factor which may influence circulating thyroid autoAbs. In fact, lithium has long been known to interact with thyroid function but the prevalence of circulating thyroid autoAbs among lithium-treated patients varies across studies (Bocchetta et al., 2016). According to Kupka et al. (2002), the prevalence of circulating thyroid autoAbs was not associated with prior lithium treatment. Indeed, TPO-Abs were found positive in 12/35 (34.3\%) patients who had never received lithium, a prevalence even higher than that found in the overall sample of BD outpatients $(64 / 226=28 \%)$. Similarly, other studies (Haggerty et al., 1997; Hornig et al., 1999; Kraszewska et al., 2015) found no significant relationship between the duration of lithium exposure and thyroid autoimmunity, while the study conducted by Cobo et al. (2015) found that the role of sex/lithium interaction on thyroid autoAbs was relevant. Several studies support the finding that female sex could represent a risk factor for an increased incidence of thyroid autoimmunity in BD (Cobo et al., 2015; Hillegers et al., 2007; Vonk et al., 2007). Conversely, other authors found no relationship between sex and the presence of thyroid autoAbs in BD patients (Hornig et al., 1999; Kraszewska et al., 2015; Kupka et al., 2002).

\subsection{Limitations}

One of the major limitations of this review is that assays, the autoAbs which were assessed, and the reporting of outcomes (for examples, dicothomous versus antibody titer, etc.) prevented us from performing a meta-analysis. Furthermore, due to the cross-sectional nature of most included studies, a causal relationship between thyroid autoimmunity and BD cannot be firmly established. The quality of included studies varied according to the NOS. Finally, the majority of previous studies assessed only the presence of TPO-Abs, providing little information regarding other thyroid autoAbs, including TG-Abs and TRAbs.

\subsection{Clinical implications}

The link between thyroid autoimmunity and BD might be relevant in clinical practice. In fact, the etiopathogenesis, clinical course, prognosis and treatment of $\mathrm{BD}$ could be influenced by an alteration in the HPT axis. This association could be compatible with an immunologic hypothesis for the development of BD. Thus, thyroid autoimmunity might represent a risk factor for a subsequent mood disorder diagnosis, at least in a subgroup of BD patients. For that reason, it could be important for the physician to monitor thyroid function carefully, mostly in depressed and mixed BD patients as well as in BD female patients.

\subsection{Research implications}

Given the potential role of thyroid autoAbs, future research should measure circulating thyroid autoAbs in patients with bipolar spectrum disorders and also in their offspring, since they might serve as a biomarker for BD (Carvalho et al., 2016). Moreover, future studies should investigate the prevalence rates of thyroid autoAbs in lithium-naïve BD patients to compare their prevalence before and after lithium treatment longitudinally. It would be interesting to assess how circulating thyroid autoAbs associate with phase and course of BD to better understand whether increased autoAbs levels occur in some subtypes of bipolar disorder, unrelated to lithium exposure. Furthermore, the identification of other specific thyroid autoAbs in different phases of BD illness merits further investigation.

\subsection{Conclusion}

Based on the results of this review, circulating thyroid autoAbs could be an independent and relevant risk factor for the pathophysiology of BD, thus representing an endophenotype for BD and a marker of vulnerability for bipolar illness.

\section{Acknowledgments}

Prof. Vieta would like to acknowledge PI 12/00912 (Spanish Ministry of Economy and Competitiveness), PN 2008-2011 (Instituto de Salud Carlos III, Subdirección General de Evaluación y Fomento de la Investigación; Fondo Europeo de Desarrollo Regional. Unión Europea, "Una manera de hacer Europa"), 2014_SGR_398 (Secretaria d'Universitats i Recerca del Departament d'Economia i Coneixement Generalitat de Catalunya).

\section{Appendix A. Supporting information}

Supplementary data associated with this article can be found in the online version at http://dx.doi.org/10.1016/j.jad.2017.06.019.

\section{References}

Azorin, J.M., Kaladjian, A., Adida, M., Hantouche, E.G., Hameg, A., Lancrenon, S., Akiskal, H.S., 2008. Factors associated with rapid cycling in bipolar I manic patients: findings from a French national study. CNS Spectr. 13, 780-787.

Barbero, J.D., Garcia-Pares, G., Llorens, M., Tost, M., Cobo, J., Palao, D., Labad, J., 2014. Thyroglobulin antibodies and risk of readmission at one year in subjects with bipolar disorder. Psychiatry Res. 219, 109-113.

Bartalena, L., Pellegrini, L., Meschi, M., Antonangeli, L., Bogazzi, F., Dell'Osso, L., Pinchera, A., Placidi, G.F., 1990. Evaluation of thyroid function in patients with rapid-cycling and non-rapid-cycling bipolar disorder. Psychiatry Res. 34, 13-17.

Bauer, M.S., Whybrow, P.C., 1986. The effect of changing thyroid function on cyclic af fective illness in a human subject. Am. J. Psychiatry 143, 633-636.

Berk, M., Kapczinski, F., Andreazza, A.C., Dean, O.M., Giorlando, F., Maes, M., Yücel, M., Gama, C.S., Dodd, S., Dean, B., Magalhães, P.V., Amminger, P., McGorry, P., Malhi, G.S., 2011. Pathways underlying neuroprogression in bipolar disorder: focus on inflammation, oxidative stress and neurotrophic factors. Neurosci. Biobehav. Rev. 35, 804-817.

Bocchetta, A., Loviselli, A., 2006. Lithium treatment and thyroid abnormalities. Clin. Pract. Epidemiol. Ment. Heal. 2, 23.

Bocchetta, A., Traccis, F., Mosca, E., Serra, A., Tamburini, G., Loviselli, A., 2016. Bipolar disorder and antithyroid antibodies: review and case series. Int. J. Bipolar Disord. 4,5 .

Boelaert, K., Franklyn, J.A., 2005. Thyroid hormone in health and disease. J. Endocrinol. $187,1-15$.

Bonnin, C.M., Martinez-Aran, A., Sanchez-Moreno, J., Torrent, C., Franco, C., Pacchiarotti, I., Vieta, E., 2010. Bipolar disorder, cognitive functioning and hypothalamic-pituitary-thyroid axis. Actas Esp. Psiquiatr. 38, 223-228.

Carta, M.G., Moro, M.F., Lorefice, L., Trincas, G., Cocco, E., Del Giudice, E., Fenu, G. Colom, F., Marrosu, M.G., 2014. The risk of bipolar disorders in multiple sclerosis. J. Affect. Disord. 155, 255-260.

Carvalho, A.F., Kohler, C.A., Fernandes, B.S., Quevedo, J., Miskowiak, K.W., Brunoni, A.R., Machado-Vieira, R., Maes, M., Vieta, E., Berk, M., 2016. Bias in emerging biomarkers for bipolar disorder. Psychol. Med. 46, 2287-2297.

Chakrabarti, S., 2011. Thyroid functions and bipolar affective disorder. J. Thyroid Res. 2011, 306367

Cobo, J., Gimenez-Palop, O., Patro, E., Perez, M., Bleda, F., Barbero, J.D., Oliva, J.C., 
Serrano, R., Berlanga, E., Garcia-Pares, G., Palao, D., 2015. Lack of confirmation of thyroid endophenotype in Bipolar Disorder Type I and their first-degree relatives. Psychoneuroendocrinology 51, 351-364.

Cowdry, R.W., Wehr, T.A., Zis, A.P., Goodwin, F.K., 1983. Thyroid abnormalities associated with rapid-cycling bipolar illness. Arch. Gen. Psychiatry 40, 414-420.

Cho, J.T., Bone, S., Dunner, D.L., Colt, E., Fieve, R.R., 1979. The effect of lithium treatment on thyroid function in patients with primary affective disorder. Am. J. Psychiatry 136, 115-116.

Eaton, W.W., Pedersen, M.G., Nielsen, P.R., Mortensen, P.B., 2010. Autoimmune diseases, bipolar disorder, and non-affective psychosis. Bipolar Disord. 12, 638-646.

Goodwin, F.K., Jamison, K.R., 2007. Manic-Depressive Illness: Bipolar Disorders and Recurrent Depression. Oxford University Press, New York.

Grande, I., Berk, M., Birmaher, B., Vieta, E., 2016. Bipolar disorder. Lancet 387, $1561-1572$

Haggerty Jr., J.J., Evans, D.L., Golden, R.N., Pedersen, C.A., Simon, J.S., Nemeroff, C.B., 1990. The presence of antithyroid antibodies in patients with affective and nonaffective psychiatric disorders. Biol. Psychiatry 27, 51-60.

Haggerty Jr., J.J., Silva, S.G., Marquardt, M., Mason, G.A., Chang, H.Y., Evans, D.L., Golden, R.N., Pedersen, C., 1997. Prevalence of antithyroid antibodies in mood disorders. Depression Anxiety 5, 91-96.

Hamdani, N., Doukhan, R., Kurtlucan, O., Tamouza, R., Leboyer, M., 2013. Immunity, inflammation, and bipolar disorder: diagnostic and therapeutic implications. Curr. Psychiatry Rep. 15, 387.

Hillegers, M.H., Reichart, C.G., Wals, M., Verhulst, F.C., Ormel, J., Nolen, W.A. Drexhage, H.A., 2007. Signs of a higher prevalence of autoimmune thyroiditis in female offspring of bipolar parents. Eur. Neuropsychopharmacol. 17, 394-399.

Hornig, M., Amsterdam, J.D., Kamoun, M., Goodman, D.B., 1999. Autoantibody disturbances in affective disorders: a function of age and gender? J. Affect. Disord. 55, 29-37.

Kraszewska, A., Chlopocka-Wozniak, M., Abramowicz, M., Sowinski, J., Rybakowski, J.K., 2015. A cross-sectional study of thyroid function in 66 patients with bipolar disorder receiving lithium for 10-44 years. Bipolar Disord. 17, 375-380.

Kupka, R.W., Nolen, W.A., Post, R.M., McElroy, S.L., Altshuler, L.L., Denicoff, K.D., Frye, M.A., Keck Jr., P.E., Leverich, G.S., Rush, A.J., Suppes, T., Pollio, C., Drexhage, H.A., 2002. High rate of autoimmune thyroiditis in bipolar disorder: lack of association with lithium exposure. Biol. Psychiatry 51, 305-311.

Kupka, R.W., Regeer, E.J., 2007. Bipolar mood disorders. Ned. Tijdschr. Geneeskd. 151, $2256-2260$

Leon-Caballero, J., Pacchiarotti, I., Murru, A., Valenti, M., Colom, F., Benach, B., Perez, V., Dalmau, J., Vieta, E., 2015. Bipolar disorder and antibodies against the N-methyld-aspartate receptor: a gate to the involvement of autoimmunity in the pathophysiology of bipolar illness. Neurosci. Biobehav. Rev. 55, 403-412.

Leyhe, T., Mussig, K., 2014. Cognitive and affective dysfunctions in autoimmune thyroiditis. Brain Behav, Immun, 41, 261-266.

Liberati, A., Altman, D.G., Tetzlaff, J., Mulrow, C., Gotzsche, P.C., Ioannidis, J.P., Clarke, M., Devereaux, P.J., Kleijnen, J., Moher, D., 2009. The PRISMA statement for reporting systematic reviews and meta-analyses of studies that evaluate healthcare interventions: explanation and elaboration. Br. Med. J. 339, b2700.

Maes, M., Bosmans, E., Calabrese, J., Smith, R., Meltzer, H.Y., 1995. Interleukin-2 and interleukin-6 in schizophrenia and mania: effects of neuroleptics and mood stabilizers. J. Psychiatr. Res. 29, 141-152.

Nielsen, C.H., Hegedus, L., Leslie, R.G., 2004. Autoantibodies in autoimmune thyroid disease promote immune complex formation with self antigens and increase B cell and $\mathrm{CD} 4+\mathrm{T}$ cell proliferation in response to self antigens. Eur. J. Immunol. 34 263-272.

Oomen, H.A., Schipperijn, A.J., Drexhage, H.A., 1996. The prevalence of affective dis order and in particular of a rapid cycling of bipolar disorder in patients with abnormal thyroid function tests. Clin. Endocrinol. 45, 215-223.

Rege, S., Hodgkinson, S.J., 2013. Immune dysregulation and autoimmunity in bipolar disorder: synthesis of the evidence and its clinical application. Aust. N.Z. J. Psychiatry 47, 1136-1151.

Sidhom, O., Laadhar, L., Zitouni, M., Ben Alaya, N., Rafrafi, R., Kallel-Sellami, M., Lahmar, H., El Hechmi, Z., Makni, S., 2012. Spectrum of autoantibodies in Tunisian psychiatric inpatients. Immunol. Investig. 41, 538-549.

Silva, L.M., Chavez, J., Canalli, M.H., Zanetti, C.R., 2003. Determination of IgG subclasses and avidity of antithyroid peroxidase antibodies in patients with subclinical hypothyroidism - a comparison with patients with overt hypothyroidism. Horm. Res. $59,118-124$.

Siwek, M., Sowa-Kucma, M., Styczen, K., Misztak, P., Nowak, R.J., Szewczyk, B., Dudek, D., Rybakowski, J.K., Nowak, G., Maes, M., 2016. Associations of serum cytokine receptor levels with melancholia, staging of illness, depressive and manic phases, and severity of depression in bipolar disorder. Mol. Neurobiol. 2016 (Epub ahead of print).

Vonk, R., van der Schot, A.C., Kahn, R.S., Nolen, W.A., Drexhage, H.A., 2007. Is autoimmune thyroiditis part of the genetic vulnerability (or an endophenotype) for bipolar disorder? Biol. Psychiatry 62, 135-140.

Wehr, T.A., Goodwin, F.K., 1979. Rapid cycling in manic-depressives induced by tricyclic antidepressants. Arch. Gen. Psychiatry 36, 555-559.

Wells, G.A., Shea, B., O'Connell, D., Peterson, J., Welch, V., Losos, M., Tugwell, P., 2014. The Newcastle-Ottawa Scale (NOS) for Assessing the Quality of Nonrandomised Studies in Meta-Analyses. University of Ottawa, Ottawa. 〈http://www.ohri.ca/ programs/clinical epidemiology/oxford.asp $>$.

Whybrow, P.C., Prange Jr., A.J., Treadway, C.R., 1969. Mental changes accompanying thyroid gland dysfunction. A reappraisal using objective psychological measurement. Arch. Gen. Psychiatry 20, 48-63. 